\title{
Planar cell polarity genes control the connectivity of enteric neurons
}

\author{
Valentina Sasselli, ${ }^{1}$ Werend Boesmans, ${ }^{1,2}$ Pieter Vanden Berghe, ${ }^{2}$ \\ Fadel Tissir, ${ }^{3}$ André M. Goffinet, ${ }^{3}$ and Vassilis Pachnis ${ }^{1,4}$
}

\begin{abstract}
1Division of Molecular Neurobiology, MRC National Institute for Medical Research, London, United Kingdom. 2'aboratory for Enteric Neuroscience, TARGID, KU Leuven, Leuven, Belgium. ${ }^{3}$ Institute of Neuroscience, Developmental Neurobiology, Université Catholique de Louvain, Brussels, Belgium. ${ }^{4}$ nstitute of Molecular Biology and Biotechnology, Foundation of Research and Technology-Hellas, Heraklion, Greece.
\end{abstract}

\begin{abstract}
A highly complex network of intrinsic enteric neurons is required for the digestive and homeostatic functions of the gut. Nevertheless, the genetic and molecular mechanisms that regulate their assembly into functional neuronal circuits are currently unknown. Here we report that the planar cell polarity (PCP) genes Celsr 3 and $F z d 3$ are required during murine embryogenesis to specifically control the guidance and growth of enteric neuronal projections relative to the longitudinal and radial gut axes. Ablation of these genes disrupts the normal organization of nascent neuronal projections, leading to subtle changes of axonal tract configuration in the mature enteric nervous system (ENS), but profound abnormalities in gastrointestinal motility. Our data argue that PCP-dependent modules of connectivity established at early stages of enteric neurogenesis control gastrointestinal function in adult animals and provide the first evidence that developmental deficits in ENS wiring may contribute to the pathogenesis of idiopathic bowel disorders.
\end{abstract}

\section{Introduction}

In the nervous system, fiber pathways often run along major developmental axes $(1,2)$. Nevertheless, the mechanisms that govern the geometric blueprint of emerging axons and their significance for connectivity and sensorimotor output remain unclear. The enteric nervous system (ENS) constitutes a network of interconnected ganglia, which are arranged radially throughout the gut and integrate local and systemic signals to control gastrointestinal motility, secretion, and blood flow (3). In vertebrates, the majority of enteric neurons and glia are derived from vagal neural crest cells which invade the foregut and, migrating rostro-caudally, uniformly colonize the entire length of the gastrointestinal tract (4). Considerable recent progress has identified a number of signaling pathways that control the migration of ENS progenitor cells and their differentiation into enteric neurons and glia (5). However, the organizing principles of enteric connectivity and the mechanisms underlying the assembly of functional circuits from differentiated enteric neurons remain unclear. This can be traced to particular challenges presented by the ENS, such as a lack of recognizable topographic organization of the plethora of neuronal subtypes and the absence of predictive rules that correlate subtype identity to postsynaptic targets. Despite the absence of an obvious anatomical and wiring logic of the ENS, enteric neuronal circuits regulate highly coordinated and stereotypic patterns of gut motility and secretion that are paramount to gastrointestinal function.

The importance of the ENS is highlighted by congenital and acquired conditions in which developmental failure (Hirschsprung disease, also referred to as HSCR) or secondary loss (e.g., Chagas disease) of enteric ganglia leads to severe gut dysmotility $(6,7)$. In addition to those conditions in which digestive abnormalities can

Authorship note: Valentina Sasselli and Werend Boesmans contributed equally to this work.

Conflict of interest: The authors have declared that no conflict of interest exists. Citation for this article: J Clin Invest. 2013;123(4):1763-1772. doi:10.1172/JCI66759. be clearly ascribed to deficits in enteric ganglia, other often debilitating disorders characterized by disturbed intestinal motor function, such as chronic intestinal pseudo-obstruction or irritable bowel syndrome, present with either inconsistent pathology of the ENS or show no changes in the number or organization of enteric ganglia $(8,9)$. Although the pathogenesis of such idiopathic bowel disorders remains poorly defined, it has been suggested that they result from subtle changes in the biochemistry or connectivity of enteric neurons, which escape the scrutiny of currently available diagnostic procedures (10). Elucidating the developmental mechanisms that underlie the connectivity of enteric neurons is therefore crucial for a broader understanding of the physiological roles of the ENS in digestion and homeostasis and for exploring the pathogenetic mechanisms of congenital or acquired idiopathic gastrointestinal motility disorders.

Here we have combined in vivo and ex vivo physiological assays with gene inactivation and single-cell labeling to demonstrate that, in mice, the planar cell polarity (PCP) pathway controls gastrointestinal function by regulating the spatial organization of neuronal processes during gut organogenesis. Our studies identify critical regulators of ENS wiring in vivo and provide insight into the connection pathology that might underlie several idiopathic gut motility disorders.

\section{Results}

Celsr 3 and $F z d 3$ are required for organization of the nascent neuronal plexus in the gut of mouse embryos. Previous studies have demonstrated that several members of the Wnt family of morphogens are expressed in the gastrointestinal tract during embryogenesis (11). Our expression analysis of genes that are known to function downstream of Wnt signaling demonstrates that Celsr3 and Fzd3 which encode, respectively, a cadherin adhesion molecule with a G-protein-coupled receptor domain (12) and a Wnt receptor (13) - are specifically expressed during embryogenesis in neuroectodermal derivatives of the gut (Figure 1). To begin exploring the potential roles of these molecules in ENS formation, we 


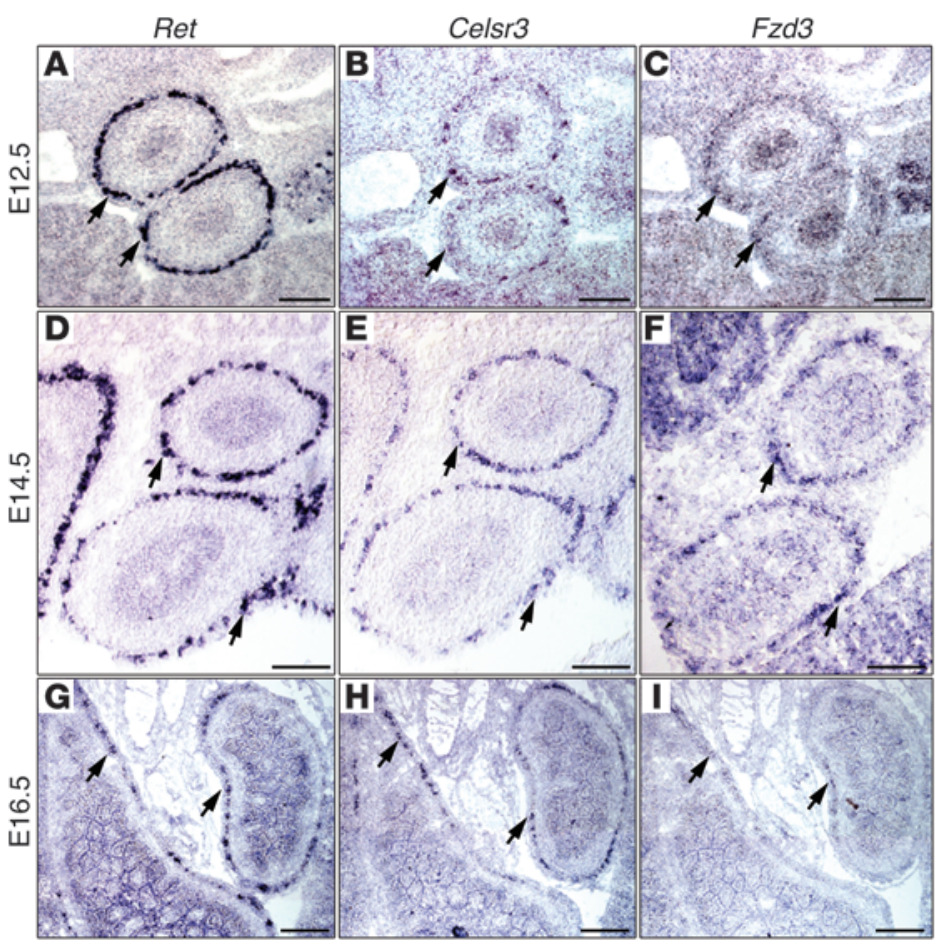

Figure 1

Celsr3 and $\mathrm{Fzd} 3$ are expressed in the developing ENS. In situ hybridization histochemistry on serial transverse sections of E12.5 (A-C), E14.5 (D-F), and E16.5 (G-I) wild-type embryos using riboprobes specific for Ret (A, D, and G), Celsr3 (B, E, and H), and Fzd3 (C, F, and I). The distribution of Celsr3 and Fzd3 transcripts within the developing gut (arrows) is very similar to that of Ret mRNA, indicating that these genes are specifically expressed by enteric neural crest derivatives. Note that Cels3 and Fzd3 appear to label only a subset of cells marked with the Ret riboprobe. Scale bars: $100 \mu \mathrm{m}$.

analyzed the nascent ENS plexus of Celsr3- and Fzd3-deficient mouse embryos $(14,15)$ using the pan-neuronal marker TuJ1 and the Wnt1-Cre/R26R-EYFP transgene combination, which drives expression of the fluorescent lineage reporter YFP in neural crest cell lineages $(16,17)$. No difference was observed in the distribution of $\mathrm{GFP}^{+}$cells and $\mathrm{TuJ} 1^{+}$neurons in the gut of control (wildtype or $\mathrm{Celsr}^{+/-}$and $\mathrm{Fzd3}^{+/-}$) and homozygous mutant embryos at E12.5 or E14.5 (Figure 2, A-C, and Supplemental Figure 1). However, when we analyzed the spatial organization of developing enteric neuronal projections, we found that the prominent $\mathrm{TuJ} 1^{+}$bundles in the midgut of E12.5 control embryos (Figure 2D) were markedly reduced in number and thickness in Celsr3 $3^{-1-}$ and $\mathrm{Fzd3}^{-/-}$littermates (Figure 2, E and F). As a consequence, the neural network in mutant samples did not display the clear longitudinal predominance that was invariably observed in wild-type samples (see Methods and Figure 2, G-I). To further characterize this phenotype, we retrogradely traced subsets of neuronal processes by DiI application on whole-mount gut preparations from E12.5 embryos. In control guts, the longitudinal processes extended for considerable distances from either side of the DiI application site and ran parallel to the long axis of the gut (Figure 2J). In addition, neuronal cell bodies were evident only on the oral side (Figure 2J), consistent with the predominantly anal direction of enteric neuronal projections at this stage (18). DiI labeling of Celsr3 $3^{-/-}$and $\mathrm{Fzd3}^{-/-}$guts identified fewer and shorter longitudinal processes (Figure 2, $\mathrm{K}$ and L), suggesting that the neurite network is disrupted. Similar defects in longitudinal tract formation and neurite organization were also observed at later developmental stages (E14.5 and P0) in both the midand hindgut of mutant embryos (Supplemental Figure 2). The severe reduction in DiI-labeled neuronal processes suggests reduced differentiation or defective neuritogenesis by immature enteric neurons. However, we found no differences in the proliferation (Supplemental Figure 3, A-D), neuronal differentiation (Supplemental Figure 3, E-H), or subtype specification (Supplemental Figure 3, I-P) of enteric neural crest cells between control and mutant embryos. Moreover, when placed in culture, mutant enteric neurons did not present any obvious defects in morphology or neuritogenesis (Supplemental Figure 4).

Celsr3 and Fzd3 specifically control the trajectory and growth of neuronal processes within the gut. To further enhance the resolution of our analysis, we introduced into the Celsr 3 and $F z d 3$ mutant backgrounds a transgenic combination (Sox10-iCreER ${ }^{T 2}$;R26R-EYFP) that drives expression of YFP in multilineage progenitors of the ENS (19). By titrating this in vivo labeling system, we were able to visualize individual $\mathrm{YFP}^{+} \mathrm{TuJ}^{+}$enteric neurons and their principal projections throughout the midgut of E12.5 control and mutant embryos and determine their organization relative to the radial and longitudinal axes of the gut (Figure 3, A and B). In control guts, the vast majority of identifiable neuronal processes were directed anally parallel to the longitudinal axis. However, in both Celsr 3 and Fzd3 mutants, a significantly larger fraction of neural projections were arranged circumferentially or directed orally (Figure 3, C-I). In addition to the altered trajectory, Celsr3- and Fzd3-deficient enteric neurons had on average shorter primary neurites (Figure 3J), while a fraction of them acquired bipolar or multipolar morphology (Figure 3K). Taken together, these experiments demonstrate that Celsr3 and $\mathrm{Fzd} 3$ control the growth and spatial organization of primary neural processes of nascent enteric neurons during development.

To determine whether the aberrant trajectory and abnormal length of neurites of Celsr3-deficient enteric neurons could be rescued by wild-type enteric neural crest derivatives, we generated Sox10-iCreER ${ }^{T 2} ; R 26 R-E Y F P$;Celsr $3^{f l-}$ embryos, which allowed the conditional (upon administration of tamoxifen) ablation of Celsr3 from a subset of neural crest progenitors and their simultaneous labeling with the fluorescent reporter YFP (20). Celsr3-deficient enteric neurons showed a significant decrease in the percentage of caudally directed neurites and a concomitant increase in circumferentially and orally projecting neurites (Figure 3, L-N). In contrast to the orientation, the length of $\mathrm{YFP}^{+} \mathrm{Celsr} 3^{-}$neurites was unaffected (Figure 3O). These experiments show that Celsr 3 is cell-autonomously required in enteric neural crest derivatives to control the spatial organization of neural projections within the gut and suggest that guidance and growth of neural processes are regulated by genetically distinct mechanisms.

Neural crest-specific inactivation of Celsr3 leads to functional abnormalities of the gastrointestinal tract. It is currently unclear whether connectivity in the ENS is based on a genetically controlled embryonic blueprint of neurite organization, or rather reflects the nonspecific adjustment of enteric circuitry to overriding functional requirements of the postnatal gut. To address this question, 

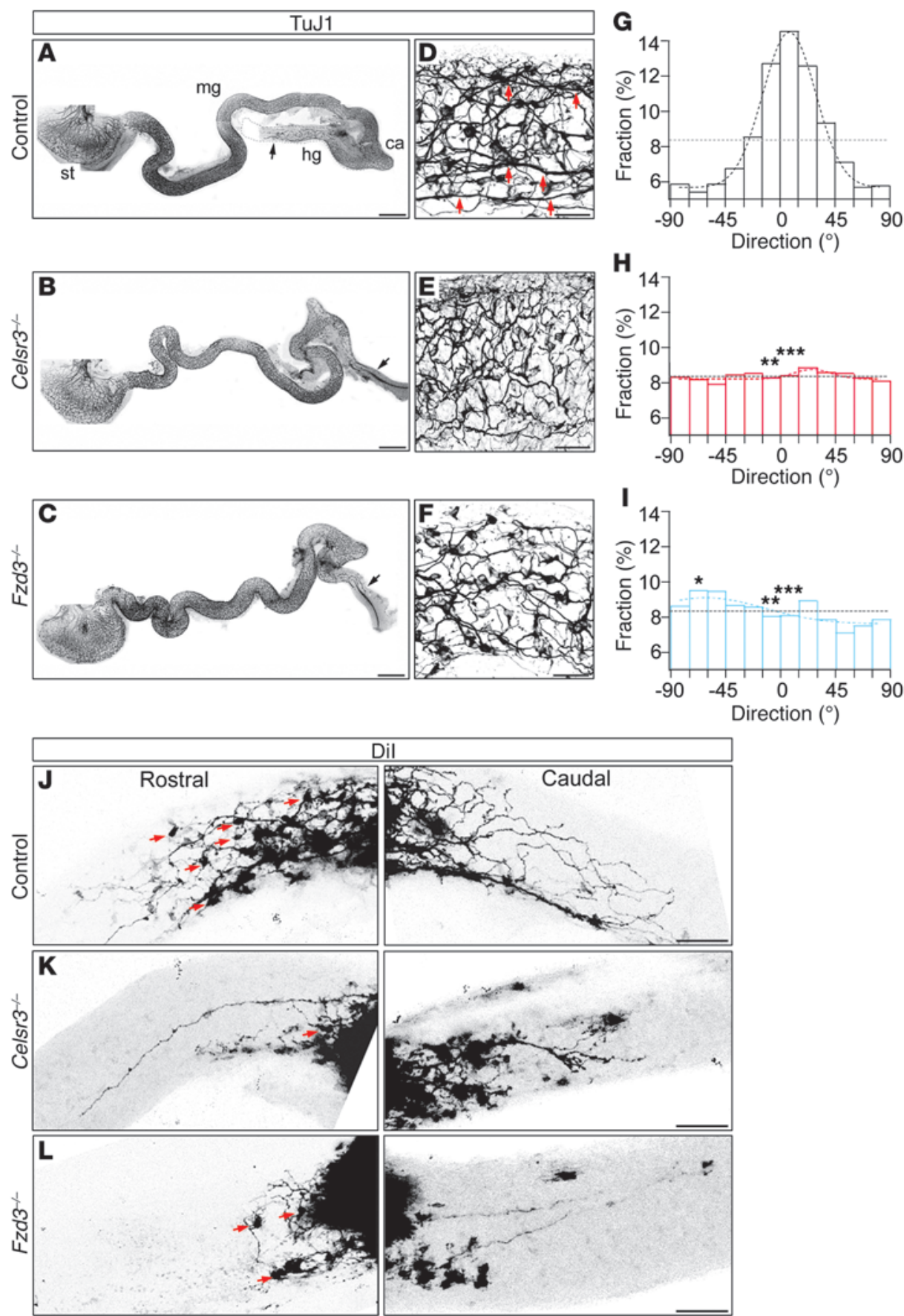

\section{Figure 2}

Deficits in the organization of neuronal processes in Celsr3 and Fzd3 mutant guts. (A-C) Grayscale inverted images of gut preparations from E12.5 control (A), Celsr3-l- (B), and $\mathrm{Fzd}^{-/-}$(C) embryos immunostained for TuJ1. Arrows indicate the position of the most caudally located neurons. (D-F) High magnification of equivalent midgut areas from preparations shown in $\mathbf{A}-\mathbf{C}$. Red arrows in $\mathbf{D}$ indicate the prominent longitudinal tracts found in control midguts, which were absent in mutant embryos. (G-I) Analysis of the distribution of the developing neuronal plexus in either one of the 12 directions $\left(15^{\circ}\right.$ wide from $-90^{\circ}$ to $\left.+90^{\circ}\right)$. The gray dotted line indicates the hypothetical case in which TuJ1+ neuronal tracts were organized randomly (equal distribution over all bins, $180^{\circ} / 12=0.083$ ). A Gaussian fit was used to identify the dominant direction in each genotype. In mutants, the plexus orientation deviates less from the random order and is significantly different from the control $(N=6$ per genotype). Two-way ANOVA, $P<0.0001$; Bonferroni's post-hoc test, ${ }^{*} P<0.05,{ }^{* *} P<0.01$, and ${ }^{* * *} P<0.001$. $(\mathbf{J}-\mathbf{L})$ Dil tracing in control $(\mathbf{J})$, Celsr ${ }^{-/-}$ (K), and $\mathrm{Fzd}^{-/-}$(L) E12.5 midguts. Left and right panels indicate the rostral and caudal sides of tracings, respectively. Note the dramatic reduction in the number and length of longitudinal projections and the number of cell bodies (arrows) in mutant preparations. ca, caecum; hg, hindgut; mg, midgut; st, stomach. Scale bars: $500 \mu \mathrm{m}(\mathbf{A}-\mathbf{C})$, $50 \mu \mathrm{m}(\mathbf{D}-\mathbf{F})$, and $100 \mu \mathrm{m}(\mathbf{J}-\mathbf{L})$. we assessed the consequences of the aberrant configuration of neurites in the gut of Celsr3- and Fzd3-deficient mouse embryos on functional output and organization of the ENS in 4-week-old animals. Since constitutive ablation of Celsr 3 and $F z d 3$ results in perinatal lethality of mice $(14,15)$, we combined conditional and null alleles of Celsr3 (Celsr $3^{f l-}$ ) with the Wnt1-Cre transgene to specifically and efficiently delete the locus in neural crest lineages, including the ENS. At weaning (P21) Celsr $3^{f l-}$;Wnt1-Cre;R26R-YFP mutant mice (referred to hereafter as Celsr $3 \mid$ Wnt 1 ) were present at the expected ratio, but their survival, size, and weight were consistently reduced relative to control littermates (Figure 4, A and $\mathrm{B})$. In addition, macroscopic examination of the gastrointes- tinal tracts of Celsr $3 \mid$ Wnt1 mice revealed abnormally contracted and dilated segments of the small bowel, localized accumulation of intestinal contents, and a higher number of smaller fecal pellets (Figure 4, C and D). This phenotype was very pronounced in moribund animals, suggesting that altered gut function contributes to the increased lethality of Celsr $3 \mid$ Wnt 1 animals. To further analyze the gastrointestinal motor activity in mutant mice, we assayed whole gut transit time with a nonabsorbable carmine red solution administered by oral gavage. Consistent with the observed abnormal gut morphology, the whole gut transit time of mutant mice was significantly slower compared with control littermates (Figure 4E). Finally, the weight and water content of 

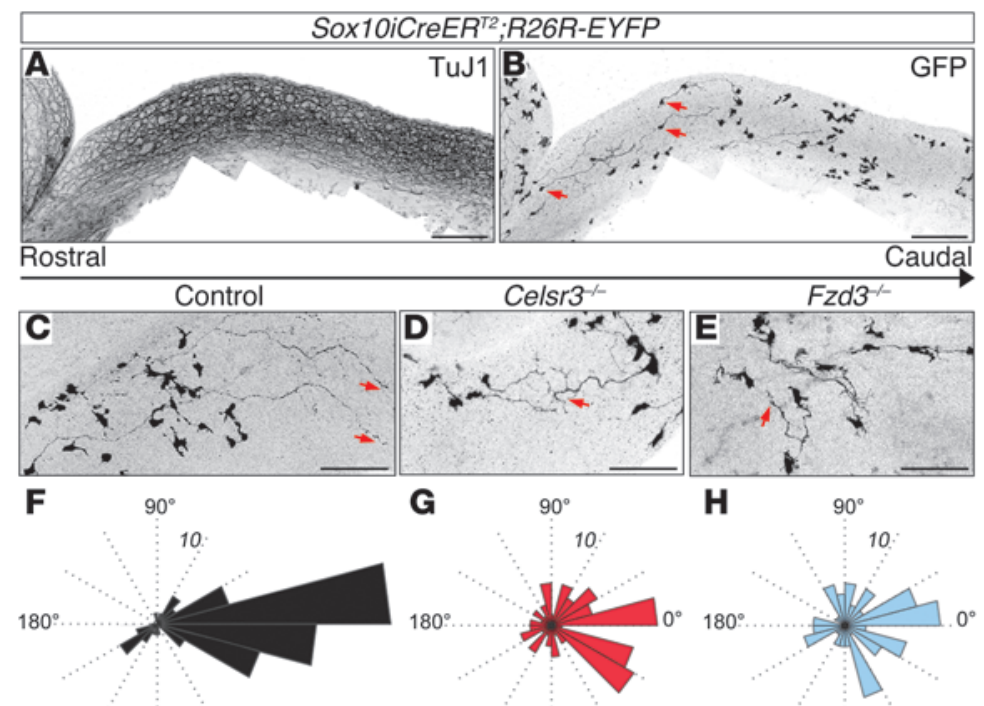

$270^{\circ}$
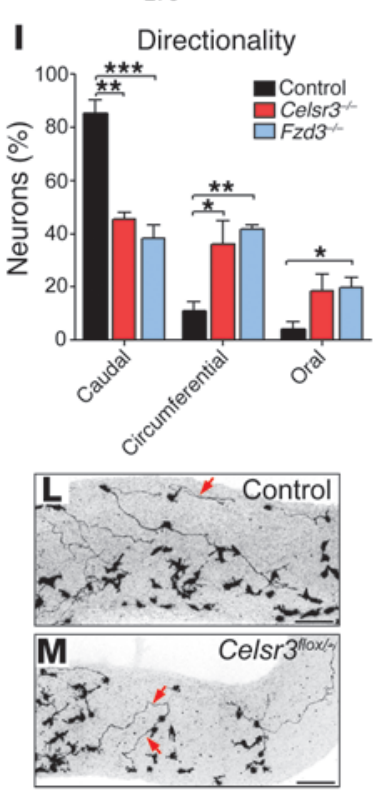

$270^{\circ}$

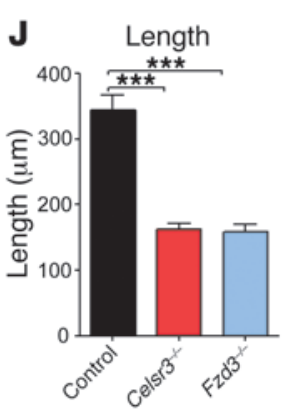

$270^{\circ}$
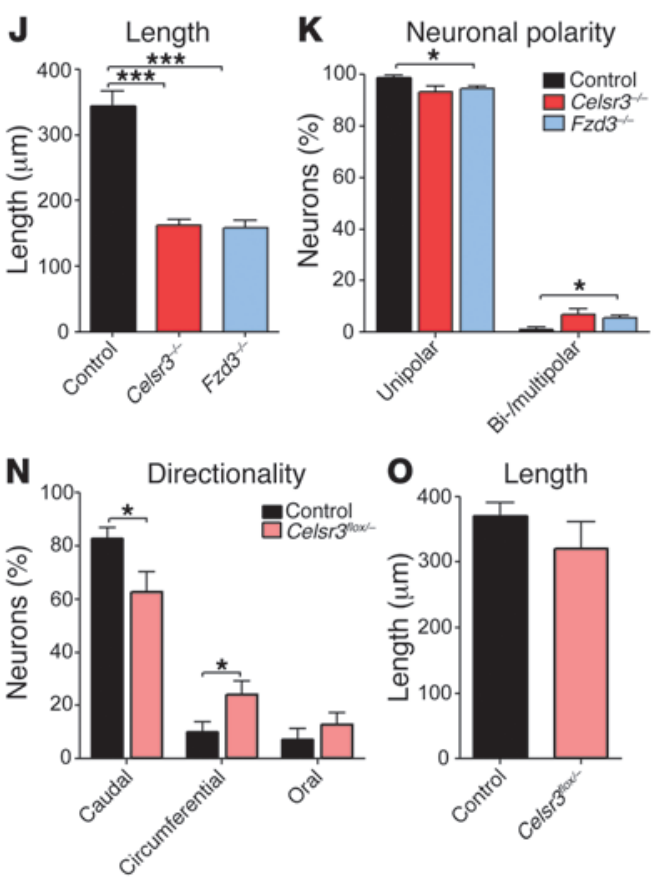

\section{Figure 3}

Celsr3 and Fzd3 are required for guidance and growth of enteric neuronal projections. (A and B) Grayscale inverted images of a gut segment from a 4-OHTexposed Sox10-iCreER ${ }^{T 2}$;R26R-EYFP embryo double immunostained for TuJ1 (A) and GFP (B). This transgenic combination allows untangling of the complex neuronal network (A) and characterization of the morphology of individual GFP-labeled neurons (B, arrows). (C-E) Representative images of GFP-labeled enteric neurons in the gut of control, Celsr3-, and Fzd3-deficient embryos. Indicated neurons project caudally (C), orally (D), or circumferentially (E). Neurons were identified and analyzed along the entire length of the midgut. $(\mathbf{F}-\mathbf{H})$ Distribution of angles formed between neurites and the longitudinal axis of the gut $(n=65)$. (I and J) Quantification of the directionality and length of neurites in the gut of control and mutant embryos. (K) Quantification of neurons with unipolar and bi-/multipolar morphology $(n \geq 80)$. One-way ANOVA, $P<0.05$; Bonferroni's post-hoc test, ${ }^{\star} P<0.05$, ${ }^{* *} P<0.01$, and ${ }^{* * *} P<0.001$. (L and $\left.\mathbf{M}\right) \mathrm{GFP}+$ neurons in $\mathrm{Celsr}^{\mathrm{fl} /+}(\mathbf{L}$, control) and Celsr $3^{\mathrm{fl}-}$ (M) embryos transgenic for Sox10-iCreER ${ }^{T 2} ; R 26 R-E Y F P$. Arrows indicate caudally (L) or circumferentially projecting (M) neurites. ( $\mathbf{N}$ and $\mathbf{O}$ ) Quantification of directionality and length of neurites in the indicated genotypes $(n \geq 107)$. Two-tailed Student's $t$ test, ${ }^{\star} P<0.05,{ }^{\star *} P<0.01$, and ${ }^{* * \star} P<0.001$. Scale bars: $200 \mu \mathrm{m}$ (A and $\mathbf{B})$, and $100 \mu \mathrm{m}$ (C-E, L, and $\mathbf{M})$. fecal pellets were consistently reduced (Figure 4, F and G). Based on these experiments, we suggest that neural crest-specific deletion of Celsr3 leads to severe gastrointestinal dysfunction.

Genetic ablation of Celsr 3 affects colonic motility. To investigate the role of Celsr3 in gastrointestinal physiology, we video recorded the spontaneous motility of colon preparations dissected from 4-week-old control and Celsr $3 \mid$ Wnt 1 mice. The resulting spatiotemporal maps allowed us to characterize the colonic migrating motor complexes (CMMCs), which constitute spontaneous ENS-mediated anally propagating contractions generated in a recurrent fashion $(21,22)$. CMMCs recorded from colons of Celsr $3 \mid$ Wnt 1 animals showed increased frequency and migrated for shorter distances relative to their counterparts in control preparations (Figure 5, A-F, and Supplemental Videos 1 and 2). Although CMMCs in Celsr 3 Wnt1 colons had a tendency to propagate more slowly, the average speed was not significantly different compared with that of controls (Figure 5G). In addition to CMMCs, control colons showed occasionally short-lived orally propagating contractions. Interestingly, such contractions were more frequent and persistent in mutant colons (Figure 5, D and $\mathrm{H}$ ). Both anally and orally propagating contractions were of neuronal origin, as they were abolished by the addition of the voltage-gated sodium channel blocker tetrodotoxin (TTX, $1 \mu \mathrm{M}$ ) (Figure 5, insets in C and $\mathrm{D}$ ). To test the propulsion of luminal contents, colonic preparations were challenged with an artificial pellet introduced into the proximal (caecal) end. In contrast to the control preparations, which reproducibly and effectively propelled the pellet toward the rectum, mutant colons repeatedly failed to generate peristalsis and often showed tonic contractile activity aborally to the luminal pellet (Figure 5, I-M, and Supplemental Videos 3 and 4). These 
A

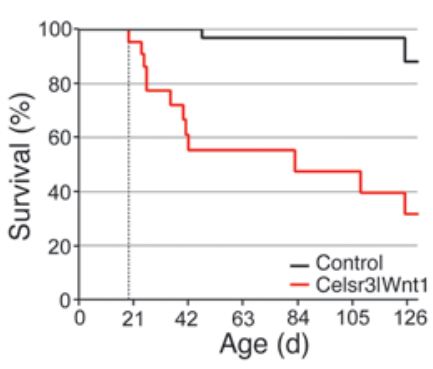

B

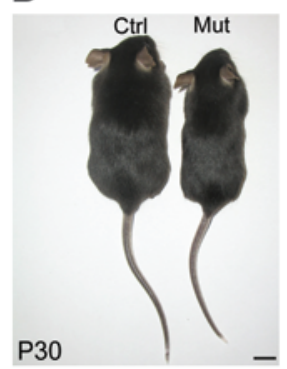

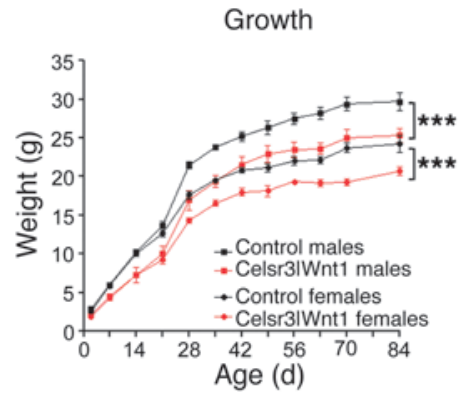

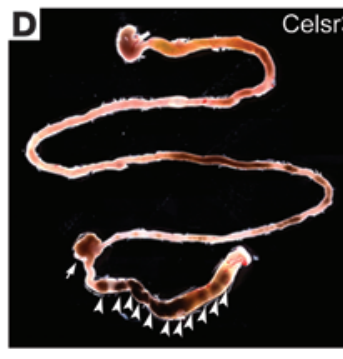

$\mathbf{F}$

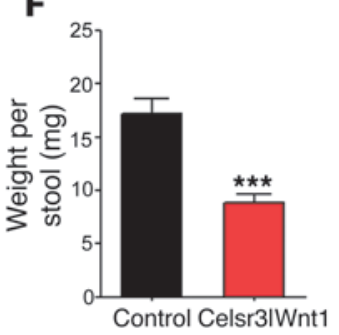

Growth

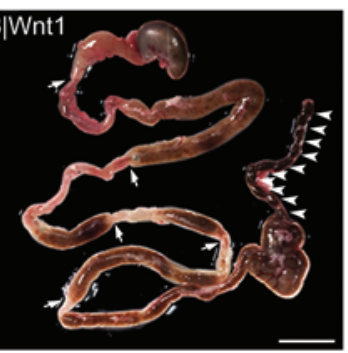

G

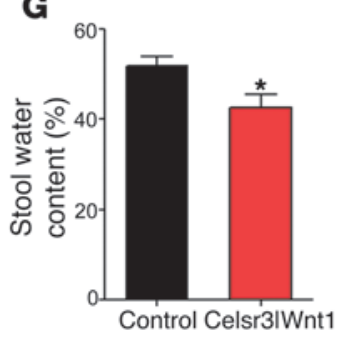

\section{Figure 4}

Neural crest-specific inactivation of Celsr3 leads to deficits in gastrointestinal function. (A) Kaplan-Meier graphs of the survival of Celsr3IWnt1 mutants and controls $(N=22$ and $N=46$, respectively). Log-rank test, $P<0.001$. (B) P30 Celsr3IWnt1 mutants (Mut) are smaller relative to wild-type littermates (Ctrl). Graphs indicating the weight increase of male and female Celsr3IWnt1 and control mice from P0-P84 ( $N \geq 3$ for each age). Two-way ANOVA, ${ }^{* * *} P<0.001$. (C and D) Photomicrographs of whole gastrointestinal tract preparations from control (C) and 2 Celsr3IWnt1 mutants (D) at P30. Mutant guts are characterized by constricted segments (arrows) which are often preceded by distended regions. Arrowheads in the colon indicate individual fecal pellets in control and mutant guts. (E-G) Gastrointestinal transit time (E) and weight of stools (F) and water content (G) of fecal pellets from control and Celsr3IWnt1 animals ( $N=15$ and $N=11$, respectively). Twotailed Student's $t$ test, ${ }^{*} P<0.05,{ }^{* *} P<0.01$, and ${ }^{* *} P<0.001$. Scale bars: $1 \mathrm{~cm}$. experiments suggest that Celsr 3 activity is required for the elaboration of enteric circuits that underlie the coordinated peristaltic activity of the gut.

Celsr 3 activity is required for organization of the neuronal plexus of the gut. Several of the features of gastrointestinal physiology identified in Celsr3-deficient animals are evocative of the uncoordinated motor activity observed in idiopathic intestinal dysmotility syndromes (23). Such syndromes, however, are associated with minimal or no changes in the organization of the enteric ganglionic plexus, thus thwarting efforts to demonstrate their neurogenic origin. Likewise, despite profound changes in the gastrointestinal physiology of Celsr 3 Wnt1 mice, no obvious abnormalities in the overall organization of the myenteric and submucosal ganglia were observed in the ileum (Figure 6, A and B) and colon (Supplemental Figure 5) of these animals. In addition, the density of enteric neurons and the relative proportion of the neuronal nitric oxide synthase-expressing (nNOS-expressing) and calretinin-expressing subtypes, which represent the two main nonoverlapping neuronal subtypes in the mouse intestine (24), were similar between control and Celsr3 $\mid$ Wnt1 mutants (Figure 6, C-E, and Supplemental Figure 5). Furthermore, we observed no changes in the network of interstitial cells of Cajal and smooth muscle layers (Figure 6, $A$ and B, and Supplemental Figures 5 and 6), consistent with the normal myogenic contractions ("ripples") recorded in mutant colon preparations (Supplemental Figure 6). Nonetheless, upon careful examination of the myenteric plexus of Celsr $3 \mid$ Wnt 1 mice, we found that the $\mathrm{TuJ} 1^{+}$interganglionic strands showed irregular trajectories and reduced thickness (Figure 6, F and G, and Sup- plemental Figure 5). DiI tracing revealed that in controls, these strands were formed by fibers extending for considerable distances from the site of dye application and were predominantly aligned with the circumferential or longitudinal axes of the gut (Figure $6 \mathrm{H}$ and Supplemental Figure 5). In Celsr $3 \mid$ Wnt1 mutants, however, DiI-labeled fibers were reduced in number and length and their trajectory did not follow a clear orientation (Figure 6I and Supplemental Figure 5). Interestingly, nNOS immunoreactivity was markedly decreased in the nitrergic interganglionic strands of Celsr3|Wnt1 mutants, whereas no difference was observed upon immunostaining for the vesicular acetylcholine transporter (vAChT) (Figure 6, J and K). This suggests that nitrergic interganglionic strands, which in the mammalian ENS are mainly formed by axons of descending interneurons and inhibitory motor neurons $(24,25)$, are selectively affected in Celsr 3 mutants. Overall, these findings argue that Celsr 3 activity is required for the organization of the enteric neuronal plexus in mammals.

\section{Discussion}

Assembly of diverse neuronal subtypes into ganglia and the growth, guidance, and branching of axons and dendrites are essential for the correct wiring of the peripheral nervous system in vertebrates. Neural crest cell migration and gangliogenesis have been studied extensively in the context of ENS development, but the mechanisms that control axon guidance and connectivity of enteric neurons remain poorly defined. Understanding such mechanisms is a crucial step for characterization of the wiring blueprint of the ENS and identification of pathogenetic cascades that lead to mam- 

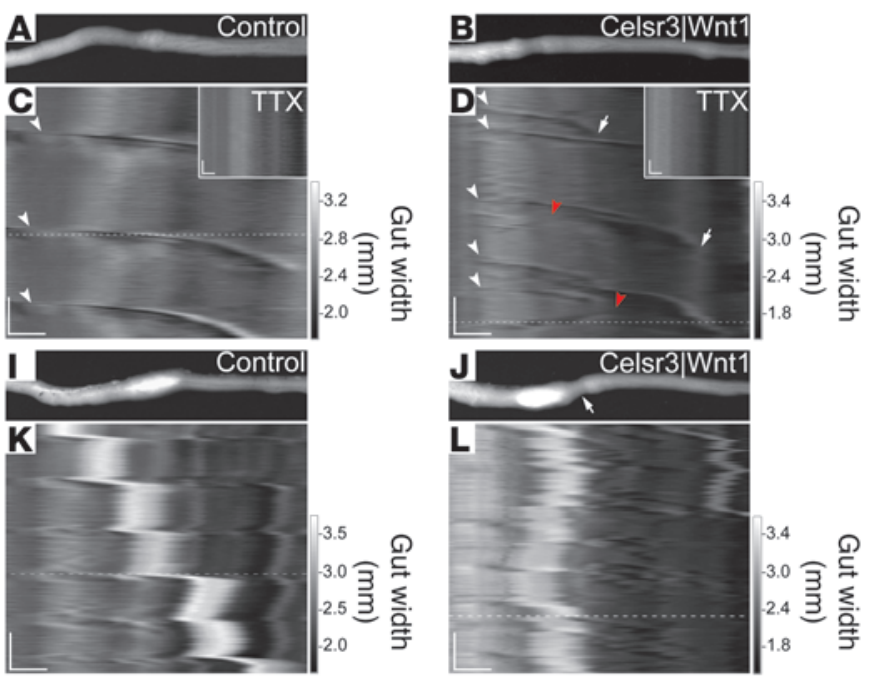
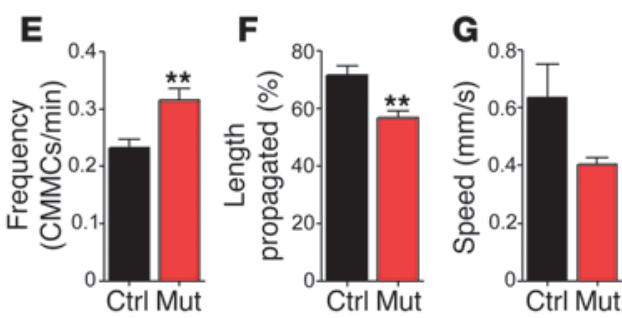

H

Frequency of oral contractions

Mut
Ctrl

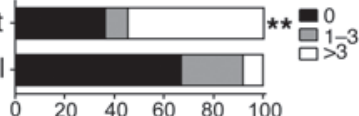

Colons (\%)

M

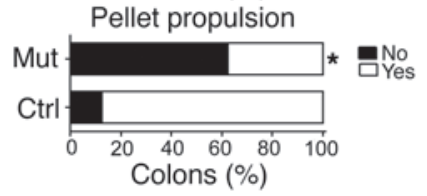

\section{Figure 5}

Severe dysmotility of the large bowel of Celsr3IWnt1 mice. (A-D) Video recordings of spontaneous motor patterns of colonic preparations from control and Celsr3IWnt1 mice were analyzed using spatiotemporal maps (C and D; see also Supplemental Videos 1 and 2). Maximum dilation (white), maximum constriction (black), and intermediate levels of constriction (grayscale) are represented over time (downward). Images in A and $\mathbf{B}$ correspond to the dashed lines in $\mathbf{C}$ and $\mathbf{D}$, respectively. CMMCs (white arrowheads) in mutant colons often stop prematurely (arrows in $\mathbf{D}$ ). Red arrowheads in $\mathbf{D}$ indicate orally directed contractions. TTX abolishes contractions (insets in $\mathbf{C}$ and $\mathbf{D})(N=3)$. ( $\mathbf{E}-\mathbf{G})$ Average frequency $(\mathbf{E})$, length propagated $(\mathbf{F})$, and speed $(\mathbf{G})$ of CMMCs $(N \geq 11)$. Student's $t$ test, ${ }^{*} P<0.05$ and ${ }^{* *} P<0.01$. (H) Proportions of preparations from control and Celsr3IWnt1 mutant mice displaying $0,0-3$, and $>3$ orally propagating contractions within a 1,000 -second recording. $\chi^{2}$ test, ${ }^{* *} P<0.01$. (I-L) Video recordings of distention-evoked motor patterns of control and Celsr3IWnt1 colons were analyzed using spatiotemporal maps (K and $\mathbf{L}$; see also Supplemental Videos 3 and 4). Images in I and $\mathbf{J}$ correspond to the dashed lines in $\mathbf{K}$ and $\mathbf{L}$, respectively. The majority of mutant colons failed to generate efficient peristalsis and often showed tonic contractions aborally to the pellet (arrow in $\mathbf{J}$ ). (M) Proportion of preparations that successfully propelled an artificial pellet. $\chi^{2}$ test, ${ }^{\star} P<0.05$. Scale bars: 150 seconds (vertical) and $5 \mathrm{~mm}$ (horizontal).

malian gut dysfunction. By combining constitutive and cell typespecific gene inactivation in mice with in vivo and ex vivo analysis of gastrointestinal physiology and high-resolution cell labeling of neural crest derivatives, we provide evidence that core components of the PCP pathway link the spatial organization of neuronal processes at early stages of enteric neurogenesis to adult gut function.

Previous studies have suggested that the longitudinal arrangement and anal orientation of enteric neuronal projections during embryogenesis is associated with, and perhaps imposed by, the rostro-caudal migration of neural crest progenitors $(18,26)$. Our present findings demonstrate that Celsr3 and Fzd3 regulate the polarized organization of nascent neurites of enteric neurons directly and cell autonomously (in the case of Celsr3), thus providing for the first time evidence for a genetic cascade that controls the blueprint of ENS wiring. Normal colonization of the gut in Celsr3- and Fzd3-deficient embryos suggests that these genes control the organization of neuronal projections independently of enteric neural crest cell migration. Nevertheless, our current data do not exclude the possibility that in addition to its effect on enteric neuronal projections, PCP signaling also regulates some aspect of enteric neural crest cell motility. Resolving this issue will require fluorescence videomicroscopy of neural crest cells in live gut preparations from wild-type and mutant embryos.

Earlier studies have demonstrated that Drosophila flamingo/starry night collaborates with frizzled to regulate PCP, a signaling pathway that aligns cells with their neighbors along body and appendicular axes $(27,28)$. In a similar manner, the mammalian orthologs of these genes, Celsr and Fzd, control the polarized organization of cells within fields of neural and non-neural tissues, often in a Wnt morphogen-dependent manner (14, 20, 29-34). Interestingly, the noncanonical Wnt5a morphogen is expressed in a graded manner along the embryonic gut (11) and is required for gut organogenesis in the context of body axis elongation $(35,36)$. These studies and our present work raise the possibility that Wnt5a, in addition to its role in intestinal organogenesis, serves as an upstream global polarizing signal for the ENS. We suggest that during enteric neurogenesis, Fzd 3 and Celsr3 function downstream of Wnt5a to link gut morphogenesis to the installation of neural circuits that control its physiological output.

Consistent with the altered arrangement of neuronal projections in Celsr3-deficient embryos, in adult conditional Celsr3 mutant mice, longitudinal interganglionic neuronal strands were often reduced in thickness and followed irregular trajectories. A major component of these tracts are axons of $\mathrm{nNOS}^{+}$ descending interneurons and inhibitory motor neurons which project caudally in the mammalian ENS $(24,25)$, suggesting that nitrergic innervation is affected in Celsr 3 mutants. This idea is further supported by the observation that genetic ablation of the $n$ Nos locus or pharmacological inhibition of its product leads to increased frequency of CMMCs $(37,38)$, a phenotype shared by the Celsr3-deficient colonic preparations. Since nNOS ${ }^{+}$enteric neurons are among the first to emerge in the ENS $(18,39)$ and their axons project longitudinally and caudally from the outset (18), we suggest that PCP signaling controls the correct spatial organization of the processes of early nitrergic neurons, which is subsequently required for the formation of functional neural cir- 
A
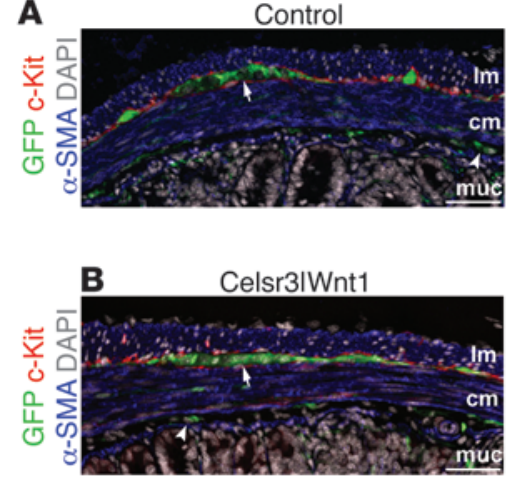
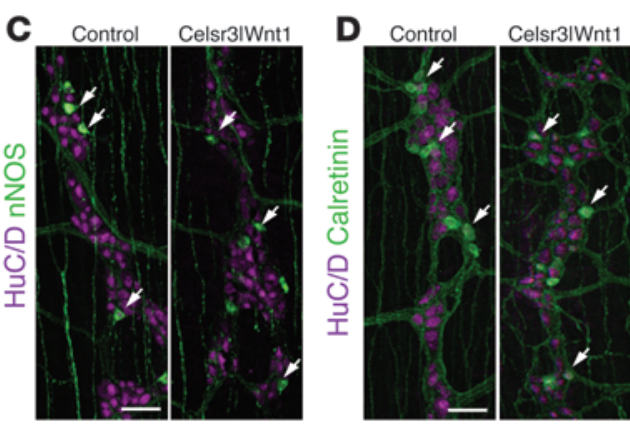

E

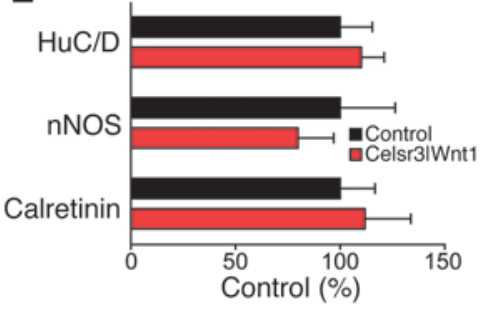

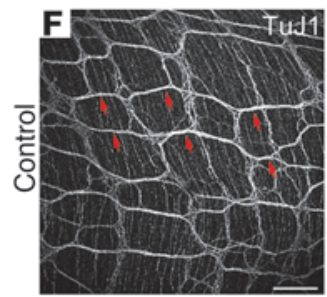
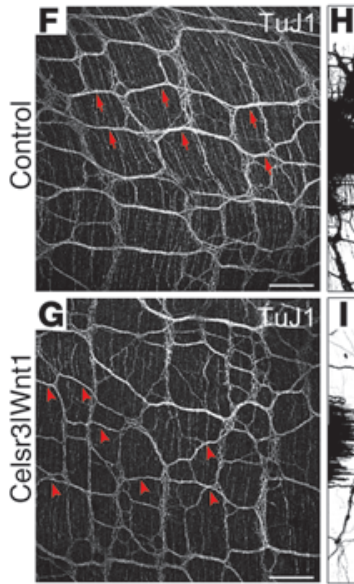
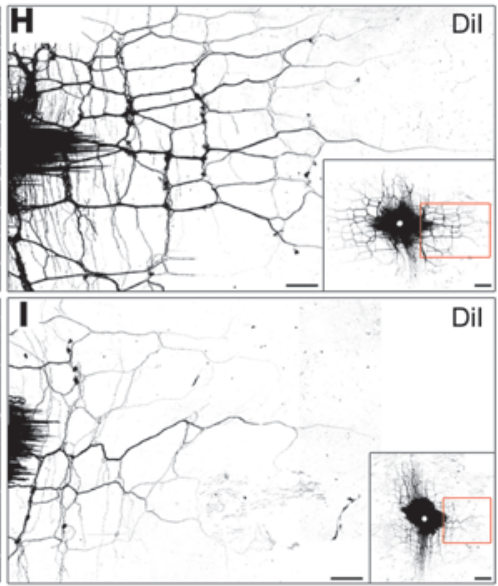
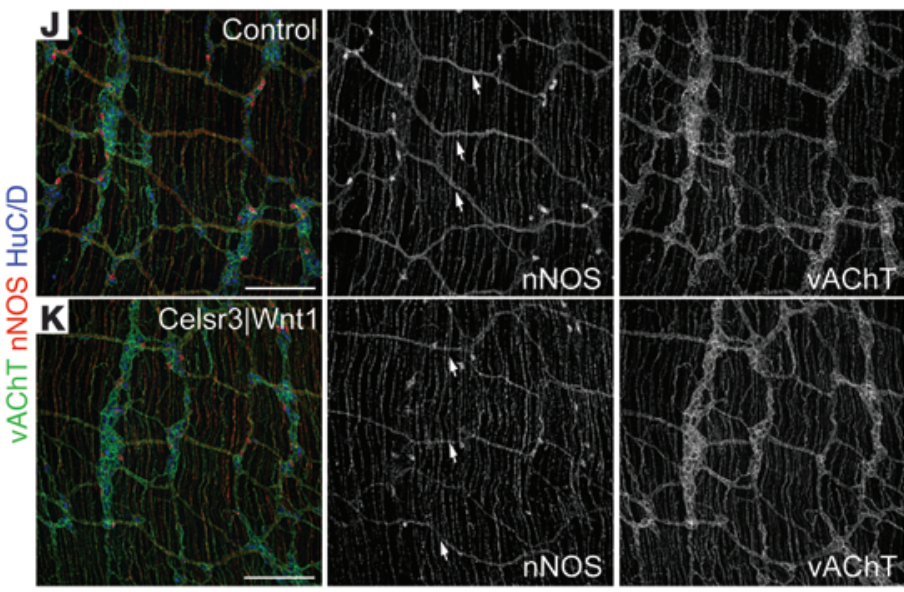

Figure 6

Subtle deficits of fiber tracts in the ENS of Celsr3IWnt1 mice. (A and B) Control and Celsr3IWnt1 ileum immunolabeled for GFP (green), c-Kit (red), $\alpha$-SMA (blue), and counterstained for DAPI (gray). (C and D) Myenteric ganglia from control and Celsr3IWnt1 mice immunostained for $\mathrm{HuC} / \mathrm{D}$ (magenta) and nNOS (green, C), or calretinin (green, D). (E) Density of HuC/D+ neurons and proportion of $\mathrm{nNOS}^{+}$and calretinin ${ }^{+}$neurons in the gut of control and Celsr3IWnt1 mice $(n \geq 1,200)$. The Student's $t$ test was nonsignificant. (F and $\mathbf{G})$ Grayscale images of myenteric plexus from control and Celsr3IWnt1 mice immunostained for TuJ1. The longitudinal trajectory of TuJ1+ interganglionic strands is often lost in Celsr3IWnt1 mutants (G). ( $\mathbf{H}$ and $\mathbf{I})$ Dil tracing of adult myenteric plexus from gut preparations that correspond to $\mathbf{F}$ and $\mathbf{G}$, respectively. Images in $\mathbf{H}$ and $\mathbf{I}$ correspond to the boxed areas of the insets. ( $\mathbf{J}$ and $\mathbf{K}$ ) Triple labeling of myenteric plexus for nNOS (red), vAChT (green), and HuC/D (blue). In mutants, longitudinal nNOS ${ }^{+}$strands were reduced in number and thickness (arrows), while $\mathrm{vAChT}^{+}$fibers were unaffected. $\mathrm{cm}$, circular muscle; Im, longitudinal muscle; mu, mucosa. Scale bars: $50 \mu \mathrm{m}$ (A-D), $200 \mu \mathrm{m}$ (F-K), and $500 \mu \mathrm{m}$ (H and I insets).

cuits. Of course we cannot exclude the possibility that Celsr3 (and Fzd3) signaling directly controls the spatial organization of neurites emerging from other neuronal subtypes or that this pathway controls other as-yet unidentified aspects of ENS connectivity at later stages. Stage- and neuronal subtype-specific ablation of the PCP genes will clarify these outstanding issues in the future. Nevertheless, our current studies indicate that relatively simple and genetically hardwired modules of neurite organization positioned at a relatively low level in the hierarchical organization of the ENS emerge during embryogenesis and serve as building blocks for the formation of higher-order neural circuits that control intestinal motility and secretion, thus providing a framework for unraveling the daunting complexity of the mammalian ENS.

Intestinal peristalsis and effective propulsion of luminal contents depend on the spatiotemporal integration of excitatory and inhibitory neural activity along the longitudinal axis of the gut. Our study provides evidence that the polarized physiological output of the ENS in adult animals depends on the asymmetric orga- nization of emerging enteric circuits during embryogenesis. In that respect, Celsr3 mutant mice represent novel genetic models in which gut motility deficits do not represent downstream secondary effects of defective gangliogenesis, as is the case for mouse strains presenting with congenital megacolon (40-43); rather, they are the direct consequence of selective and subtle alterations of ENS wiring. Gastrointestinal motility disorders, including intestinal pseudo-obstruction and functional bowel disorders, are often associated with dysmotility, changes in transit time, and uncoordinated propulsive activity $(23,44)$. Although such conditions often lead to serious disability, no clear evidence of pathological changes in neuronal or non-neuronal gut tissues has been consistently observed in these cases $(9,45)$. Nevertheless, the clinical presentation of these conditions is indicative of altered ENS function and suggests that at least some of these cases might result from subtle abnormalities in the underlying enteric circuitry. Based on the evidence we present here, we suggest that changes in the activity of conserved PCP genes in humans lead to 
aberrant organization and connectivity of the ENS and represent a plausible pathogenetic mechanism for some debilitating idiopathic bowel disorders.

\section{Methods}

Animals. The generation of the Celsr3- (14), Celsr $3^{\text {flox }}$ (20), and Fzd3- (15) alleles, and the Wnt1-Cre (16), Sox10iCreER ${ }^{T 2}$ (19), and R26R-EYFP (17) transgenes in mice have been described previously. In Celsr $3^{f l-; W n t 1-C r e ~(C e l s r 3 \mid W n t 1) ~}$ animals, the Rosa26R-EYFP reporter was used to monitor Cre activity. For labeling of individual enteric neurons in vivo, 4-hydroxytamoxifen (4-OHT; Sigma-Aldrich) was dissolved in an ethanol/sunflower oil (1:9) mixture at $10 \mathrm{mg} / \mathrm{ml}$ and administered intraperitoneally $(3 \mu \mathrm{g} / \mathrm{g})$ to E10.5 pregnant females transgenic for the Sox $10 i \mathrm{CreER}^{T 2} ; R 26 R-Y F P$ reporter system. For all embryonic studies, the day of the vaginal plug was considered E0.5.

In situ bybridization. In situ hybridization histochemistry was performed essentially as described (46) on sections from E12.5, E14.5, and E16.5 embryos. The generation of riboprobes specific for Fzd3, Celsr3 (47), and Ret (48) have been reported previously.

Gastrointestinal transit time. Total gastrointestinal transit time was measured as previously described (49). Animals were placed individually in cages devoid of bedding, and after fasting for 1 hour, $0.3 \mathrm{ml}$ of $6 \%(\mathrm{w} / \mathrm{v})$ carmine red dye (Sigma-Aldrich) in $0.5 \%(\mathrm{w} / \mathrm{v})$ methylcellulose (Sigma-Aldrich) was administered to each mouse by oral gavage. The time period from gavage until the emergence of the first red-colored pellet was recorded as total intestinal transit time. Hereafter, animals were returned to their home cage. Maximum observation time was 5 hours and in all animals analyzed, a red-colored stool was observed within this time frame.

Stool analysis. Average total weight per stool was measured by placing individual animals in a novel cage and collecting all stools produced over a 1-hour period $(9-10 \mathrm{am})$. Stools were then allowed to desiccate at $75^{\circ} \mathrm{C}$ overnight to determine their dry weight. Water content per stool was calculated as the difference between wet and dry weight expressed as a percentage.

Live video imaging and spatiotemporal mapping of colonic motility. Ex vivo video imaging and analysis of colonic motility was performed as described previously $(37,50)$. Entire colons were carefully isolated and loosely pinned in an organ bath chamber, continuously superfused (flow rate: $4 \mathrm{ml}$ per minute) with oxygenated $\left(95 \% \mathrm{O}_{2}\right.$ and $\left.5 \% \mathrm{CO}_{2}\right)$ Krebs solution (in mM: $120.9 \mathrm{NaCl}, 5.9 \mathrm{KCl}, 1.2 \mathrm{MgCl}_{2}, 2.5 \mathrm{CaCl}_{2}, 1.2 \mathrm{NaH}_{2} \mathrm{PO}_{4}, 14.4$ $\mathrm{NaHCO}_{3}, 11.5$ Glucose) kept at $37^{\circ} \mathrm{C}$. Intestines were allowed to equilibrate for 45 minutes, after which movies of colonic motility were captured (2.5 Hz frame rate) with a QICAM-Fast camera using QCapture Pro 6.0 software (Q-Imaging). Images were read into an Igor Pro (WaveMetrics) and analyzed using custom-written algorithms. The bowel's edges were determined and the width computed and mapped over time. From the generated spatiotemporal maps, the frequency, travel distance, and speed (measured as the average speed over the total propagated distance) of propagating contractions were determined. For analysis of colonic propulsion, an artificial pellet was inserted into the colon, $0.5-1 \mathrm{~cm}$ starting from the proximal (caecal) end. Propagation of the pellet was recorded and analyzed as described above.

Adult myenteric plexus preparation. Preparations of adult gut muscle strips with adherent myenteric plexus were obtained by placing 2-cm-long pieces of small intestine and colon (prewashed in PBS) over a 1-ml pipette. A small incision was made longitudinally on the outer muscle layer and a cotton swab soaked in PBS was used to strip away the longitudinal and circular muscle layers and the adherent myenteric plexus from the underlying mucosa. Tissue strips were then stretched and pinned flat onto a dish coated with Sylgard 184 Elastomer (Dow Corning) using stainless steel 0.2-mm insect pins (Agar Scientific) and processed for immunofluorescence staining or DiI labeling.
DiI labeling. Adult myenteric plexus preparations and embryonic guts were fixed in $4 \%(\mathrm{w} / \mathrm{v})$ PFA and $0.1 \%(\mathrm{w} / \mathrm{v})$ EDTA in PBS at $4{ }^{\circ} \mathrm{C}$ for 30 minutes and 2 hours, respectively. Tissues were anchored to Sylgard-coated (Dow Corning) dishes using $0.2-\mathrm{mm}$ insect pins by pinning the edges of the muscle strips (adult preparations) or the stomach and caecum (embryonic guts) to the dish. An additional pin that was dipped in DiI tissue-labeling paste (Life Technologies) was applied in the central region of the peel or midgut. Tissues were covered in $4 \%$ PFA and $0.1 \%$ EDTA in PBS and placed at $37^{\circ} \mathrm{C}$ for 7 days. After the incubation period, tissues were washed and mounted in PBS and promptly examined using a TCS SP5 confocal microscope (Leica).

Cell culture. For "short-term cultures" E12.5 guts were washed in $\mathrm{Ca}^{2+}$ and $\mathrm{Mg}^{2+}$-free PBS (Life Technologies) and digested for 5 minutes with $1 \mathrm{mg} / \mathrm{ml}$ collagenase/dispase solution (Roche) at $37^{\circ} \mathrm{C}$. Tissues were washed in $\mathrm{PBS}$ and further dissociated into a single-cell suspension by pipetting in Opti-MEM (Life Technologies). After a brief centrifugation, the entire cell suspension was plated onto fibronectin-coated ( $20 \mu \mathrm{l} / \mathrm{ml}$; Sigma-Aldrich) 8-well chamber slides (Thermo Fisher Scientific) in Opti-MEM supplemented with L-glutamine (1 mM; Life Technologies) and a penicillin/streptomycin antibiotic mixture (100 U/ml; Life Technologies). For cell proliferation analysis, $10 \mu \mathrm{M}$ EdU (Invitrogen) was added to the culture medium. Cultures were maintained for up to 4 hours in an atmosphere of $5 \% \mathrm{CO}_{2}$.

For cultures of primary neurons ("long-term cultures"), E12.5 guts were dissociated as described for short-term cultures. After centrifugation, cells were resuspended in neurobasal medium (Life Technologies) supplemented with B27 and N2 supplements (Life Technologies), L-glutamine $(1 \mathrm{mM})$, and a penicillin/streptomycin antibiotic mixture $(100 \mathrm{U} / \mathrm{ml})$, then plated onto poly-D-lysine ( $1 \mathrm{mg} / \mathrm{ml}$; Sigma-Aldrich) and laminin-coated $(1 \mu \mathrm{g} / \mu \mathrm{l}$; Sigma-Aldrich) 8-well chamber slides. Neuronal cultures were maintained for 4 days in an atmosphere of $5 \% \mathrm{CO}_{2}$.

Immunofluorescence. Immunofluorescence of dissociated gut cultures and neuronal cultures was carried out as reported previously (51). EdU detection was performed by using the Click-iT EdU Alexa Fluor 594 Imaging kit (Invitrogen) followed by an immunofluorescence protocol. Adult tissues were fixed in 4\% PFA in PBS for 30 minutes at room temperature, whereas embryonic guts were fixed for 2 hours at $4^{\circ} \mathrm{C}$. Samples were blocked in PBS containing $10 \%$ heat-inactivated sheep serum (Biosera) and $0.1 \%$ TritonX100 (Sigma-Aldrich). Primary antisera used in the study are as follows: rabbit anti- $\alpha$-SMA (1:1000; Abcam), rabbit anti-calretinin (1:500; Swant), goat anti-c-Kit (1:500; R\&D Systems), rabbit anti-nNOS (1:200; Life Technologies), mouse anti-TuJ1 (1:1,000; Covance), and guinea pig anti-vAChT (1:250; Life Technologies). Rabbit anti-GFP (1:1,000; Life Technologies) and rat anti-GFP (1:1,000; Nacalai Tesque) antibodies were used to detect expression of the YFP reporter, and the 2 terms are used interchangeably. Primary antibodies were applied overnight at $4{ }^{\circ} \mathrm{C}$. Secondary antibodies were goat or donkey hosted Alexa Fluor 488-, Alexa Fluor 568-, and Cy5-conjugated anti-mouse, anti-goat, anti-rabbit, and anti-rat, and Alexa Fluor 488 anti-guinea pig (all 1:500; Life Technologies and Jackson ImmunoResearch) were applied for 2 hours at room temperature. After antibody treatment the samples were mounted with mounting media containing DAPI (Vectashield or Prolong Gold; Invitrogen).

Image acquisition and analysis. Microphotographs of gastrointestinal tracts were acquired using a QICAM-Fast camera and QCapture Pro 6.0 software (both from Q-Imaging). Images of fluorescence samples (immunolabeled and DiI) were acquired with a TCS SP5 laser confocal microscope (Leica) using standard excitation and emission filters for visualizing DAPI, Alexa Fluor 488, Alexa Fluor 568, DiI, and Cy5.

All fluorescence images were processed with Adobe Photoshop CS2 (Adobe Systems), while analyses were performed using the image processing package Fiji/ImageJ (Wayne Rasband, NIH). Analysis of neuronal morphology in vitro was performed by using the plug-in for ImageJ, NeuronJ (52). 
To analyze whether the developing neuronal plexus forms in an ordered fashion, and whether its spatial organization displays a dominant direction, we used the plug-in for ImageJ, Directionality (Jean-Yves Tinevez). First, merged confocal stacks of midgut regions immunolabeled for TuJ1 were blinded and oriented in the $0^{\circ}$ direction (longitudinal axis of the gut) and Fourier transformed. After applying a 12-bin spatial filter in Fourier space, the amount of power per bin was calculated. These values represent how much spatial information is repeated along either one of 12 directions $\left(15^{\circ}\right.$ wide from $-90^{\circ}$ to $\left.+90^{\circ}\right)$. A Gaussian fit was used to identify the dominant direction and indicates how much this distribution deviated from a random order, in which case all information would be equally distributed over each of the 12 bins.

Directionality of projections in Sox $10-i C r e E R^{T 2} ; R 26 R-E Y F P$ guts was defined by the angle formed to the longitudinal axis of the gut with a line connecting the region of the cell body where the processes emerged to the end of their tip. Rose plots showing the distribution of angles were generated in MATLAB (MathWorks). For analysis of directionality, angle measurements were divided into 3 bins: caudal for angles ranging from $335^{\circ}$ to $45^{\circ}$ (counterclockwise); circumferential from $45^{\circ}$ to $135^{\circ}$ and from $225^{\circ}$ to $315^{\circ}$; and oral from $135^{\circ}$ to $225^{\circ}$.

Statistics. Cell counting was performed on a total sample of cells specified as " $n$ " and obtained from at least 3 independent embryos or adult animals per genotype. Physiological measurements were obtained from a number of independent animals indicated as " $N$." Differences between datasets were determined either by log-rank test (for survival curves), 2-tailed Student's $t$ test, and ANOVA followed by a Bonferroni's post-hoc test (for single and multiple comparison tests, respectively), or $\chi^{2}$ test (for proportions). A $P$ value less than 0.05 was considered to be significant. Significant differences in $P<0.05, P<0.01$, and $P<0.001$ are indicated with *, **, and ${ }^{* *}$, respectively. Statistical analysis was performed with Microsoft Excel (Microsoft) and GraphPad Prism software (GraphPad Software). All graphs error bars represent the standard error of the mean (SEM). Nonsignificant differences (ns) were $P>0.05$.

Study approval. All experimental procedures with mice were approved by the ethical review panel of the National Institute for Medical Research and were carried out under the authority of a United Kingdom Home Office Project License.

\section{Acknowledgments}

We thank the members of the Pachnis lab for critical comments on the manuscript and useful discussions. We would also like to thank Roberto De Giorgio for insightful comments on the manuscript. This work was supported by the Medical Research Council. W. Boesmans was supported by a postdoctoral fellowship from FWO (Research Foundation Flanders, Belgium).

Received for publication September 12, 2012, and accepted in revised form January 14, 2013.

Address correspondence to: Vassilis Pachnis, Division of Molecular Neurobiology, MRC National Institute for Medical Research, The Ridgeway, Mill Hill, London NW7 1AA, United Kingdom. Phone: 442088162113; Fax: 442088162109; E-mail: vpachni@ nimr.mrc.ac.uk.
1. Ramon y Cajal S. Histology of the Nervous System. New York, New York, USA: Oxford University Press; 1911.

2. Wedeen VJ, et al. The geometric structure of the brain fiber pathways. Science. 2012;335(6076):1628-1634.

3. Furness JB. The Enteric Nervous System. Hoboken, New Jersey, USA: Wiley-Blackwell; 2006.

4. Heanue TA, Pachnis V. Enteric nervous system development and Hirschsprung's disease: advances in genetic and stem cell studies. Nat Rev Neurosci. 2007;8(6):466-479.

5. Sasselli V, Pachnis V, Burns AJ. The enteric nervous system. Dev Biol. 2012;366(1):64-73.

6. Amiel J, et al. Hirschsprung disease, associated syndromes and genetics: a review. J Med Genet. 2008; 45(1):1-14.

7. Chuenkova MV, Pereiraperrin M. Neurodegeneration and neuroregeneration in Chagas disease. Adv Parasitol. 2011;76:195-233.

8. De Giorgio R, Cogliandro RF, Barbara G, Corinaldesi R, Stanghellini V. Chronic intestinal pseudo-obstruction: clinical features, diagnosis, and therapy. Gastroenterol Clin North Am. 2011; 40(4):787-807.

9. Longstreth GF, Thompson WG, Chey WD, Houghton LA, Mearin F, Spiller RC. Functional bowel disorders. Gastroenterology. 2006;130(5):1480-1491.

10. Gershon MD. Developmental determinants of the independence and complexity of the enteric nervous system. Trends Neurosci. 2010;33(10):446-456.

11. Lickert H, Kispert A, Kutsch S, Kemler R. Expression patterns of Wnt genes in mouse gut development. Mech Dev. 2001;105(1-2):181-184.

12. Nakayama M, Nakajima D, Nagase T, Nomura $N$, Seki N, Ohara O. Identification of high-molecular-weight proteins with multiple EGF-like motifs by motif-trap screening. Genomics. 1998;51(1):27-34

13. Yang-Snyder J, Miller JR, Brown JD, Lai CJ, Moon RT. A frizzled homolog functions in a vertebrate Wnt signaling pathway. Curr Biol. 1996;6(10):1302-1306.

14. Tissir F, Bar I, Jossin Y, De Backer O, Goffinet AM. Protocadherin Celsr3 is crucial in axonal tract development. Nat Neurosci. 2005;8(4):451-457.
15. Wang Y, Thekdi N, Smallwood PM, Macke JP, Nathans J. Frizzled-3 is required for the development of major fiber tracts in the rostral CNS. J Neurosci. 2002;22(19):8563-8573.

16. Danielian PS, Muccino D, Rowitch DH, Michael SK, McMahon AP. Modification of gene activity in mouse embryos in utero by a tamoxifen-inducible form of Cre recombinase. Curr Biol. 1998; 8(24):1323-1326.

17. Srinivas $S$, et al. Cre reporter strains produced by targeted insertion of EYFP and ECFP into the ROSA26 locus. BMC Dev Biol. 2001;1:4.

18. Young HM, Jones BR, McKeown SJ. The projections of early enteric neurons are influenced by the direction of neural crest cell migration. J Neurosci. 2002; 22(14):6005-6018.

19. Laranjeira C, et al. Glial cells in the mouse enteric nervous system can undergo neurogenesis in response to injury. J Clin Invest. 2011;121(9):3412-3424.

20. Zhou L, et al. Early forebrain wiring: genetic dissection using conditional Celsr3 mutant mice. Science. 2008;320(5878):946-949.

21. Bush TG, Spencer NJ, Watters N, Sanders KM, Smith TK. Spontaneous migrating motor complexes occur in both the terminal ileum and colon of the C57BL/ 6 mouse in vitro. Auton Neurosci. 2000; 84(3):162-168.

22. Fida R, Lyster DJ, Bywater RA, Taylor GS. Colonic migrating motor complexes (CMMCs) in the isolated mouse colon. Neurogastroenterol Motil. 1997; 9(2):99-107.

23. Stanghellini V, et al. Natural history of chronic idiopathic intestinal pseudo-obstruction in adults: a single center study. Clin Gastroenterol Hepatol. 2005; 3(5):449-458.

24. Qu ZD, Thacker M, Castelucci P, Bagyanszki M, Epstein ML, Furness JB. Immunohistochemical analysis of neuron types in the mouse small intestine. Cell Tissue Res. 2008;334(2):147-161.

25 . Brookes SJ. Classes of enteric nerve cells in the guinea-pig small intestine. Anat Rec. 2001;262(1):58-70.

26. Hao MM, Young HM. Development of enteric neu- ron diversity. J Cell Mol Med. 2009;13(7):1193-1210.

27. Adler PN. Planar signaling and morphogenesis in Drosophila. Dev Cell. 2002;2(5):525-535.

28. Zallen JA. Planar polarity and tissue morphogenesis. Cell. 2007;129(6):1051-1063.

29. Seifert JR, Mlodzik M. Frizzled/PCP signalling: a conserved mechanism regulating cell polarity and directed motility. Nat Rev Genet. 2007;8(2):126-138.

30. Jones C, Chen P. Planar cell polarity signaling in vertebrates. Bioessays. 2007;29(2):120-132.

31. Gray RS, Roszko I, Solnica-Krezel L. Planar cell polarity: coordinating morphogenetic cell behaviors with embryonic polarity. Dev Cell. 2011; 21(1):120-133.

32. Copp AJ, Greene ND. Genetics and development of neural tube defects. J Pathol. 2010;220(2):217-230.

33. Devenport D, Fuchs E. Planar polarization in embryonic epidermis orchestrates global asymmetric morphogenesis of hair follicles. Nat Cell Biol. 2008; 10(11):1257-1268.

34. Sugimura R, et al. Noncanonical wnt signaling maintains hematopoietic stem cells in the niche. Cell. 2012;150(2):351-365.

35. Cervantes S, Yamaguchi TP, Hebrok M. Wnt5a is essential for intestinal elongation in mice. Dev Biol. 2009;326(2):285-294.

36. Matsuyama M, Aizawa S, Shimono A. Sfrp controls apicobasal polarity and oriented cell division in developing gut epithelium. PLoS Genet. 2009; 5(3):e1000427.

37. Roberts RR, Murphy JF, Young HM, Bornstein JC. Development of colonic motility in the neonatal mouse-studies using spatiotemporal maps. Am J Physiol Gastrointest Liver Physiol. 2007; 292(3):G930-G938.

38. Dickson EJ, Heredia DJ, McCann CJ, Hennig GW, Smith TK. The mechanisms underlying the generation of the colonic migrating motor complex in both wild-type and nNOS knockout mice. Am J Physiol Gastrointest Liver Physiol. 2010;298(2):G222-G232.

39. Branchek TA, Gershon MD. Time course of expression of neuropeptide $\mathrm{Y}$, calcitonin gene-re- 
lated peptide, and NADPH diaphorase activity in neurons of the developing murine bowel and the appearance of 5-hydroxytryptamine in mucosal enterochromaffin cells. J Comp Neurol. 1989; 285(2):262-273.

40. Hosoda K, et al. Targeted and natural (piebald-lethal) mutations of endothelin-B receptor gene produce megacolon associated with spotted coat color in mice. Cell. 1994;79(7):1267-1276.

41. McCallion AS, Stames E, Conlon RA, Chakravarti A. Phenotype variation in two-locus mouse models of Hirschsprung disease: tissue-specific interaction between Ret and Ednrb. Proc Natl Acad Sci U S A. 2003;100(4):1826-1831.

42. Southard-Smith EM, Kos L, Pavan WJ. Sox10 mutation disrupts neural crest development in Dom Hirschsprung mouse model. Nat Genet. 1998; 18(1):60-64.

43. Uesaka T, Nagashimada M, Yonemura S, Enomoto H. Diminished Ret expression compromises neuronal survival in the colon and causes intestinal aganglionosis in mice. $J$ Clin Invest. 2008; 118(5):1890-1898.

44. Manabe N, Wong BS, Camilleri M, Burton D, McKinzie S, Zinsmeister AR. Lower functional gastrointestinal disorders: evidence of abnormal colonic transit in a 287 patient cohort. Neurogastroenterol Motil. 2010;22(3):293-e82.

45. Di Nardo G, et al. Review article: molecular, pathological and therapeutic features of human enteric neuropathies. Aliment Pharmacol Ther. 2008; 28(1):25-42.

46. Schaeren-Wiemers N, Gerfin-Moser A. A single protocol to detect transcripts of various types and expression levels in neural tissue and cultured cells: in situ hybridization using digoxigenin-labelled cRNA probes. Histochemistry. 1993;100(6):431-440.

47. Tissir F, Goffinet AM. Expression of planar cell polarity genes during development of the mouse CNS. Eur J Neurosci. 2006;23(3):597-607.
48. Pachnis V, Mankoo B, Costantini F. Expression of the c-ret proto-oncogene during mouse embryogenesis. Development. 1993;119(4):1005-1017.

49. Nagakura Y, Naitoh Y, Kamato T, Yamano M, Miyata K. Compounds possessing 5-HT3 receptor antagonistic activity inhibit intestinal propulsion in mice. Eur J Pharmacol. 1996;311(1):67-72.

50. Hennig GW, Costa M, Chen BN, Brookes SJ. Quantitative analysis of peristalsis in the guinea-pig small intestine using spatio-temporal maps. J Physiol. 1999;517(pt 2):575-590.

51. Bondurand N, Natarajan D, Thapar N, Atkins C, Pachnis V. Neuron and glia generating progenitors of the mammalian enteric nervous system isolated from foetal and postnatal gut cultures. Development. 2003;130(25):6387-6400.

52. Meijering E, Jacob M, Sarria JC, Steiner P, Hirling $H$, Unser M. Design and validation of a tool for neurite tracing and analysis in fluorescence microscopy images. Cytometry A. 2004;58(2):167-176. 